\title{
What role should an IACUC play when a PI's manuscript is on the line?
}

\author{
Jerald Silverman, DVM
}

\begin{abstract}
"The political reality is that there is significant pressure on the IACUCs not to question the research protocols." So wrote Joan Schaffner in 2010 (ref. 1). But IACUC questioning of his research protocol was the reality facing Dr. Bennett Rothman, an assistant professor of neurophysiology at Great Eastern University. A journal manuscript authored by Rothman was returned for modifications. The recommendation was to repeat one of his experiments with male rabbits as the original submission used only females and a reviewer suggested that Rothman should determine if there was a sex-related component to his findings. Rothman knew that this publication was important to his career and although he was confident that his results were independent of sexual influences, he nevertheless submitted an amendment to his
\end{abstract}

IACUC protocol to repeat the experiment, using male rabbits.

The amendment reviewer was Dr. Linda Myles, the same person who was a designated reviewer of Rothman's original submission. At the time of the initial submission Myles had questioned the use of only female rabbits, and Rothman provided what she considered to be a reasonable explanation of why both sexes were not needed. Now that Rothman requested to redo his experiment with male animals, Myles asked for an explanation and Rothman's response was that he had no choice but to repeat the study in order to get his work published. Myles then requested full committee review of the amendment as she was not convinced that Rothman provided an adequate explanation for repeating the study. This was not acceptable to Rothman because he had a time limit to resubmit his paper and his amendment would not even be discussed by the full committee for more than a month. Desperate for help, Rothman contacted the IACUC chairman to plead his case for a rapid review. The chairman was sympathetic but said that his hands were tied by federal regulations and there was nothing he could do to expedite the process.

Given the circumstances facing Rothman, would you have called for full committee review? Should the IACUC chairman have done anything to hasten the review of the protocol? Do you think that the manuscript reviewer's recommendation was an adequate justification for redoing the experiment?

1. Schaffner, J. An Introduction to Animals and the Law (Springer, New York, 2010).

\section{RESPONSE}

\section{Teamwork can go a long way}

Chris Boehm \& Myriam Casillas

As there seems to be no guidance in the Animal Welfare Act (AWA) or the Public Health Service (PHS) policy, Rothman is indeed in a conundrum. However, there are a number of ways to work within the system without knowing more about his research.

New NIH guidelines have been recently published regarding sex differences in preclinical research studies for guidance ${ }^{1}$. Although the main thrust of the new guidelines seems to be aimed at including more females in research studies, the policy was intended to ensure that both males and females are included despite the overreliance on males in the past. Thus, without knowing more about the type of research Rothman engages in, the manuscript reviewer was justified in asking for the experiment to be performed in males.

As well, the IACUC chairman could call an emergency meeting of the IACUC to discuss this issue as time was crucial and there is no rule preventing an 'extra' meeting from occurring. IACUC Committees can meet as often as necessary to fulfill their responsibility ${ }^{2}$. It is also not clear if this IACUC has a designated member review system, based on the fact that Myles escalated the amendment to the full committee. Using a designated member review system in the future may prevent the necessity of emergency IACUC meetings, as having an IACUC meeting for every 'emergency' may prove to be overwhelming to the IACUC members and administration, and also promotes deviation from IACUC deadlines and guidelines. Even though the intentions are good, it could set a bad precedent by calling for an emergency meeting in this particular case.
There is no guideline or rule that prevents the IACUC from reviewing the science in animal protocols. However, acceptable practice discourages such a review. Rothman could ask his first reviewer, Myles, for help on justifying the inclusion of male rabbits in his amendment, as Myles must have had a good reason to ask for the male rabbits during the first review. Rothman could have worked closely with Myles to discuss the options before sending the amendment to full committee and thereby increasing the administrative burden for others. If the IACUC is uncertain if the male rabbits are justified, the IACUC is well within its bounds to ask for input from outside experts or call for a formal scientific merit review ${ }^{2}$, although this could possibly slow the project and manuscript down even more.

Finally, it may be wise for the Great Eastern IACUC to discuss and recommend a policy on acceptable justification, controls, and current guidelines to deal 